\title{
Peripheral nerve bionic interface: a review of electrodes
}

\author{
Christopher Russell ${ }^{1}$ Aidan D. Roche ${ }^{2,3} \cdot$ Samit Chakrabarty $^{1}$ (D)
}

Received: 21 November 2018 / Accepted: 11 February 2019 / Published online: 22 February 2019

(c) The Author(s) 2019

\begin{abstract}
As the demand for sensory feedback to and from prosthetic limbs becomes increasingly desirable, implantable neural interfaces are becoming more attractive. Here, we briefly review the current landscape of extra-neural electrodes for interfacing the peripheral nervous system exploring both clinical and exploratory sciences.
\end{abstract}

Keywords Peripheral nervous system $\cdot$ Neural electrode $\cdot$ Prothesis

\section{Introduction}

Neural electrodes for electrical interfacing with the body have been used for a range of treatments of chronic conditions requiring electrical recording, stimulation to excite or inhibit the target organ (Cogan 2008). These treatments offer selectivity and tunability of the electrical interfaces allowing optimisation of the therapy specific to the individual's needs. Further, this electroceutical treatment is a welcomed alternative to pharmaceutical options for the same conditions to avoid unwanted side effects as well as added specificity of the delivery (Mishra 2017).

This same technology has been evolving significantly in prosthetic technologies to the point where sensory feedback is now highly desirable. This has stemmed from the rapid development of implantable neural interface technologies that provide electrical interfaces with the nervous system either directly to the cortex (Schwartz et al. 2006) and/or to the peripheral nervous system (PNS) (Dhillon et al. 2004). This is to supersede the indirect sensory feedback methods for prosthetic such as electro-tactile stimulation, vibrotactile stimulation and modality-matched feedback which currently lack selectivity and stability (Ghafoor et al. 2017)

Samit Chakrabarty

S.Chakrabarty@leeds.ac.uk

1 School of Biomedical Sciences, Faculty of Biological Sciences, University of Leeds, Leeds LS2 9JT, UK

2 Sheffield Teaching Hospitals NHS Foundation Trust, Sheffield S10 2JF, UK

3 ECAT, College of Medicine, The University of Edinburgh, Edinburgh EH16 4TJ, UK and control of the prosthetic by myoelectric control (Jiang et al. 2012).

The peripheral nerves (Fig. 1a) act as a conduit made of mixed nerve fibres which are in turn made of axons of sensory or motor neurones. These are arranged in groups called fascicles surrounded by a sheath that holds them in bundles, and then finally a fascial layer that surrounds the whole nerve itself. When making any device used to interface with the PNS, this anatomical complexity needs to be considered, particularly the interaction of electrodes with the nerve's structures. Peripheral nerves are bidirectional pathways, communicating signals for both sensation and motor commands to and from the spinal cord and the periphery. In severe peripheral nerve injuries, these pathways can be blocked or lost, resulting in loss of function (Seddon 1942). Alternatively, as in the case of amputations, these neural pathways may be intact but with both a loss of sensory inputs and muscular targets (Dhillon et al. 2004). Bioengineered neuroelectronic interfaces, together with surgical techniques and appropriate prostheses, may provide a means to overcome these deficits (Roche et al. 2014).

Traditionally, neural electrodes have been used in laboratory settings to investigate the properties of nerves or their cortical and spinal relationships (Stein et al. 1975). As the understanding of peripheral nerves has increased and concurrent technologies have advanced, neural electrodes are beginning to enable the development of clinically applicable neuroelectronic interfaces (Rutten 2002). As the goal of these interfaces is to provide a medium to replace the loss of both sensory and motor pathways, electrodes must be selectively considered for their stimulating and recording capabilities. For example, in the case of nerve palsies 


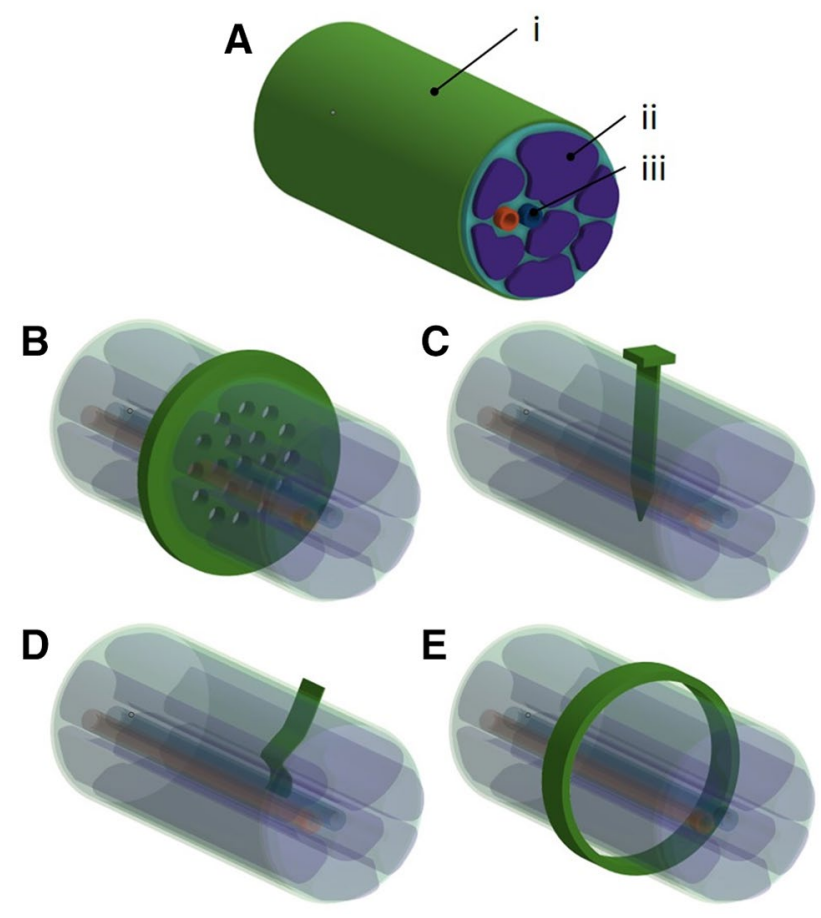

Fig. 1 a Simplified schematic of the peripheral nerve; (i) epineurium, (ii) fascicle containing axons and (iii) blood vessels. Representative placement of the $\mathbf{b}$ regenerative, $\mathbf{c}$ intra-fascicular, $\mathbf{d}$ inter-fascicular and e extra-neural electrode for electrical interfacing with the PNS (electrical tethering omitted from diagrams)

appropriate electrical stimulation can be used to elicit a targeted muscular response, such as the use of functional electrical stimulation (FES) in foot drop (Lyons et al. 2002), bladder control in paraplegia (Brindley 1994; Brindley et al. 1982), or treatment of epilepsy by vagal nerve stimulation (Ben-Menachem 2002). Additionally, direct recording of motor action potentials of nerves can be used for prosthetic control in amputees (Navarro et al. 2005). However, with increasingly sophisticated EMG decomposition methods, the latter may soon be unnecessary (Amsuess et al. 2016).

Where directly interfacing with peripheral nerves may become the most useful, is the direct stimulation of sensory pathways. A recent study of the brachial plexus and its derivative nerves in the human arm has shown that sensory axons outnumber motor axons by a ratio of 9:1 (Gesslbauer et al. 2017). Clearly, sensory inputs have a profound contribution to overall motor control and being able to replace these inputs through direct electrical stimulation could alter the way patients are treated and prostheses are designed.

Recent studies in upper limb amputees have shown that percutaneous cuff or intrafascicular electrodes can coarsely stimulate the sense of touch when coupled with a prosthetic limb (Raspopovic et al. 2014; Rossini et al. 2010). These studies have been demonstrated to work in both the laboratory setting, as well as in temporary home testing kits
(Graczyk et al. 2018; Tan et al. 2014). While these proofs of concepts are encouraging, concerns remain over percutaneous systems for long term use due to the risk of infections, wound breakdown and material failure (Pannek et al. 2005). Wireless systems may be more appropriate if the technical challenges of power delivery and biocompatibility can be overcome. A successful example of transcutaneous interfacing with the nervous system is the use of cochlear implants to overcome hearing loss. As a mature technology with proven clinical results, it demonstrates that sensory deficits can be treated with appropriate neuroelectronic interfacing (Wilson et al. 1991).

While this review focuses on peripheral nerve interfacing, it is important to note that recent work in paraplegic patients with spinal cord injuries has demonstrated that selective recording and subsequent stimulation of motor pathways can enable patients to regain the ability walk (Angeli et al. 2018; Gill et al. 2018; Wagner et al. 2018). The latter work particularly demonstrates that lower motor neurone function is not lost after spinal cord injury, but rather is dormant, and with appropriate neuroelectronic interfacing can be reactivated for voluntary control. This could have potential implications on neuroelectronic interface designs that harness central signals to stimulate peripheral nerves, thus bypassing the zone of injury.

In this review, we explore peripheral electrode designs of the cuff type, addressing many aspects of the system. This is to emphasize the complexity of using implantable electrodes for advancing smart prostheses by highlighting many areas of research such as the power transfer and communication techniques to the choice of materials. We also address current commercial options of these electrodes before discussing our perspective on peripheral neural interfaces for smart prostheses.

\section{Electrode types}

The long held view of implantable technologies is the more sensitive and selective neural electrodes are the more invasive electrodes (Hoffmann et al. 2006). This has been accepted for PNS electrodes which can be viewed as being owed to the distance between the electrode interfaces and the axons; as a result, this can influence threshold currents when stimulating axons. Surgically implanted neural interfaces for the PNS can be categorized as the following (Fig. 1), starting with the more invasive methods (Adewole et al. 2016);

- Regenerative electrodes: these utilise sieve electrodes which are a mesh of electrical contacts (Lago et al. 2005). The nerve bundle is transected, and realigned either side of the sieve. Aided by neural growth, axons reconnect 
through the electrically connected sieve allowing both stimulation and recording of signals.

- Intra-fascicular electrodes: these utilise penetrating electrodes which are pushed into the nerve bundle, and directly into/through the fascicles. Electrodes such as silicon needles (McDonnall et al. 2004), transverse intrafascicular multichannel electrode (TIME) (Boretius et al. 2010) and longitudinal intra-fascicular electrode (LIFE) (Yoshida et al. 2000) have all demonstrated reliable interfacing with the PNS.

- Inter-fascicular electrodes: similar to intra-fascicular electrodes, these electrodes penetrate the epineurium, but not the perineurium; the electrodes occupy the space between the fascicles allowing the central axons to be recorded/stimulated (Tyler and Durand 1997).

- Extra-neural electrodes: these are the least invasive electrodes and are commonly referred to as cuff electrodes. The PNS remains intact, with an electrode placed on the outer surface of the nerve, outside the epineurium.

Extra-neural electrodes are the preferred choice of electrodes in this work due to being less traumatic than the other alternative methods listed, and therefore avoid loss of functionality due to the biological response to the trauma owed to the implantation procedure. At this point, it is worth noting that for short-term applications, a recent group of transient technologies has become evident (Bettinger 2018; Koo et al. 2018). These implants are constructed from a library of materials that are readily absorbed into the body such as silk and polyurethanes for insulation and magnesium for electrical conductors. They are designed to dissolve post-implantation after a period has elapsed removing the need for surgery to remove the implant. Whilst it is worth noting that these bio-resorbable technologies exist, they are not suited for long term applications which are desired for prosthetic limb control/feedback.

\section{Electrode design}

Exploratory sciences have more freedom to explore and exploit the technologically evolving neural interfaces. The electrode development has moved beyond the scope of electronic engineers and into the development of new materials and design concepts, commanding researches from biology, chemistry, physics, computer sciences and many more. In exploratory studies, and where sufficient engineering resources are available, the electrodes are bespoke to the research question. Consequently, the mechanical construct of extra-neural electrodes takes several forms, each requiring their own methods of fixing the electrodes into position.
- Split-cylinder cuff electrodes have a tubular structure which is opened along the long axis, allowing the electrode to be placed around the nerve. The tube is then sutured closed using either embedded sutures, eyelets or an interlocking tube (Haugland 1996). The electrical interface contacts are on the inside surface of the tube and can be concentric or along the axis of the cuff. The size of the cylinders is predefined at the fabrication stage to suit the target application.

- Spiral cuff electrodes rely on the elasticity of the cuff electrode to wrap itself around the target nerve. The elasticity of the cuff allows self-sizing of the implant around different nerve diameters, adapts to swelling of nerves, and a faster placement of the electrodes when compared to split-cylinder type.

- Composite flat interface nerve electrode (C-FINE) are similar to split-cylinder cuff electrode are sutured shut around the target nerve, but have soft compression and reshape the nerve from cylindrical to elongated oval (Charkhkar et al. 2018; Freeberg et al. 2017). These semi-rigid FINE electrodes compress the axons closer to the electrical contacts but chronic damage to the axons are a concern. Developers of these technologies have argued FINE electrodes are better than the cylindrical cuff electrode by allowing the nerves to swell (Tyler and Durand 2002).

- Flexible neural clip (Lee et al. 2017) are planar-polyimide based electrodes designed to mechanically clip onto the nerves. These electrodes are limited to spacing electrodes along the long axis of the nerve. However, they do offer a quick implantation of the electrode around the target nerve.

- Flexible split ring electrode are similar to the flexible neural clip, but are positioned perpendicularly to the long axis of the nerve (Lee et al. 2017). Although, located at a single position along the long axis of the nerve it addresses multiple radial positions.

- Neural ribbon electrode are wrapped helically around the nerve, away from an anchor point sutured into position (Xiang et al. 2016).

- Zip-tie like designs using parylene materials have an interlocking design allowing the electrode to be tied around the nerves without the need to tie in sutures (Cobo et al. 2017; Yu et al. 2014).

- Nano-clip use a 3D printed clip to press two carbon nanotube electrodes against the nerves (Lissandrello et al. 2017). These are small in volume, and only require access to one side of the PNS; all other designs listed require access around the PNS.

The selection of the electrode type reflects the required positions of the electrode around the nerves whether concentric around the nerve or positioned along the long axis of 
the nerve. Along the nerve allows for propagation measurements, whereas concentric contacts will address different groupings of axons. With respect to selectivity of the cuff electrodes, where the electrode dimensions and shape are scrutinised, a smaller contact area would be more spatially selective but have a higher electrical impedance. Electrical contacts for neuroscience applications have since been engineered to increase the effective surface area to reduce the electrical impedance (and increase the charge injection capacity) by roughening the surface through electroplating (Cui and Martin 2003) or nano-patterning (Schlie-Wolter et al. 2013).

A second criterion is to address the speed and ease of implantation. Reducing the time performing surgery will reduce the time under anaesthesia which may lead to faster recovery (Russell et al. 2019). Furthermore, the anchoring of the implant remains critical, as migration of the electrodes may necessitate surgical repositioning or new implantation (Eldabe et al. 2015). Incidentally, there is no preferred method of anchoring implants into position and remains to be "based on personal experience [of the surgeon] or theoretical concepts that make intuitive sense, but have not been actually tested in vivo" (Eldabe et al. 2015). However, anchoring to the nerves are not without challenges as the nerves need to be allowed to swell as well as move freely around the neighbouring soft tissue (skin, muscle), preventing these sites being used as anchor points (Boretius et al. 2010; Naples et al. 1988).

Once implanted, the longevity and stability of the implant become critical. Encapsulation materials which are conventionally electrical insulators need to be both biocompatible and resistant to bio-dissolution (Geddes and Roeder 2003; Joung 2013). Cuff electrode based implants have used flexible materials such as silicone (polydimethylsiloxane; PDMS) (Ordonez et al. 2014), parylene (Yu et al. 2014) and polyimide (PI) (Stieglitz et al. 2000). Material selection has reflected the design of the implant, with thin planar design using PI and parylene, whereas thicker devices have used PDMS, and combinations of multiple materials have also been demonstrated. These materials will also be assessed based on their chemical and mechanical similarity to the target tissue to prevent an undesirable immune response to these alien materials (Guo 2016). An additional method of reducing the foreign body response is to incorporate eluting chemicals such as anti-inflammatory agents which are released in the surrounding tissue post-implantation (Cheung 2007). Alternatively, modifying the surface morphology has been shown to influence the protein and cell behaviour (Ereifej et al. 2013a, b). This can be applied to all electrode designs.

The electrical interface must be stable as a foreign body response over the contacts within excitable tissue will increase the separation between the electrode and the target tissue, leading to a raised stimulation threshold that is proportional to the scar tissue thickness and a drop in recording sensitivities (Geddes and Roeder 2003). Consequently, there have been several studies exploring different metals such as gold and platinum, as well as different surface morphologies (Schlie-Wolter et al. 2013). Further improvement of the longevity and stability of the electrodes have come from subsequent coatings of platinum-iridium, iridium oxide, carbon nanotubes, hydrogels, conducting polymers such as Polypyrrole/polystyrenesulfonate (PPy/PPS) (Cui and Martin 2003) and poly(ethylene dioxythiophene) (PEDOT). However, for some of these coatings, in particular conducting polymers, there is an additional technological challenge of delamination of these films from the underlying conductors (Guo 2016).

Whilst the material options appear to only be constricting the choices of exposed materials (encapsulants and electrical contacts), the choice of enclosed materials should be noted. A common cause of implant failure is a breakdown of the outer layer of the implant either through delamination, insulation leakage or cracks, therefore the use of potentially toxic materials should be avoided where possible throughout the implant design regardless of their initial physical exposure (Barrese et al. 2013).

Alternatively, the foreign body response can be anticipated and overcome by altering the operating parameters. Cardiac pacemakers' threshold current is established during surgery, then doubled to account for the expected bioresponse to the implant (Geddes and Roeder 2003). The longevity of the implant can be further improved through the operation of the electrodes, stimulating without a DC bias will reduce electro-decomposition of the electrical contacts, whilst bi-phasic pulses with a zero-net-charge have a similar response (Geddes and Roeder 2003).

Wired tethers are used to communicate the electrical signals to and from the PNS electrodes. To get these signals out of the body to the external hardware under chronic conditions, either a percutaneous, transcutaneous connector or wireless communication is used. An established norm for non-permanent electrical tethering, especially in exploratory sciences is to use an Omnetics ${ }^{\mathrm{TM}}{ }^{\circledR}{ }^{\circledR}$ micro-/nano-connectors or head-stages fixed into place on the skull, while other groups choose to use percutaneous wires without intermittent electrical interconnects (Schiefer et al. 2016). However, these interfaces have a continuous risk of infection which has "caused many to conclude that fully implantable, telemetered systems are necessary" for long term prosthetic applications (Schultz and Kuiken 2011). Transcutaneous connections through wireless transdermal communications where capacitive, inductive, acoustic and optical methods have been proposed, offering a non-destructive and non-disruptive communication through the tissues (Teshome et al. 2018). However, for wireless systems to be fully implantable 
for long-term chronic applications, an added complication of powering the implant needs to be addressed; batteries offer only a time limited solution necessitating further surgery. Current approaches include wireless power transfer (Agarwal et al. 2017) and energy harvesting (Mitcheson 2010) but currently lack the power transfer rates needed for the increasing volumes of communicated data and rates along with stimulation power of implantable technologies.

As the number of channels increase, along with a demand for higher temporal resolution in the data, the size of the connectors will inherently increase to impractical dimensions further endorsing the use of multiplexed channels on a high data rate communication channel offered by the wireless systems. Currently, a number research teams are exploring these aspects of signal processing and development of signal amplification hardware for front-end electronics to compress and stream signals out.

For chronic applications, which is expected for long-term prosthetic use, the overall design should be suited for sterilization to destroy any biological agents on the surface of the implants, to prevent infections. These may include established methods such as dry heat, electron beam- and gammairradiation, ethylene oxide, steam (autoclave), hydrogen peroxide, formaldehyde sterilization and ozone, as well as new methods such as vaporized peracetic acid, high intensity or pulsed light and microwave radiation (Joung 2013; Tipnis and Burgess 2018). The selection of the sterilization method reflects material choice of the implants with polymers; e.g. gamma irradiation is fast and efficient, but can degrade polymers through "cross-linking, chain scission or a combination of both" (Tipnis and Burgess 2018).

\section{Commercial extra-neural electrodes for PNS}

Despite there being significant strides in electrode design options, commercially available designs are relatively limited (see Table 1). The PNS electrodes on offer are of split-cylinder and spiral design using silicone or PI host substrates. The market has established a norm of using platinum, leaving the end-user to select their electrodes based on the electrode channel count and the design of the cuffs.

For clinical applications, technologies need to be CE marked for European market, or FDA approved (US market). Of the commercial electrodes listed in Table 1, of which a majority are US based suppliers, none of the products are FDA approved preventing clinical application of these technologies, thus affecting development of clinically suited prosthetic limb technologies. These implantable electrodes, carry the highest risk when compared with other medical technologies, are class III devices and therefore have stringent approval process including a premarket approval to weigh the health benefits against the risk (Tipnis and Burgess 2018).

A potential route around the FDA approval is to explore Investigational Device Exemptions (IDEs) where allowing data to be collected for understanding the associated risk; the IDE application must show that the potential benefits outweigh the risks. IDEs require the end users to seek approval for specific applications. This limits the appropriateness of repurposing technology for a different application unless it has already been stated and reviewed. Therefore, using an existing technology for a purpose which differs from the designed application, should be avoided. Humanitarian device exemption is another FDA approved route for marketing healthcare technologies in the US with a removed "requirement for a clinical trial

Table 1 Comparison of commercial peripheral nerve electrodes

\begin{tabular}{|c|c|c|c|c|c|c|}
\hline Company & Website & Substrate & Interface material & Thickness & Channels & Approval \\
\hline NeuroNexus & neuronexus.com & PI & $\mathrm{Pt}$ & $20 \mu \mathrm{m}$ & $3-24$ & Not FDA approved \\
\hline MicroProbe & microprobes.com & $\begin{array}{l}\text { Silicone }(\text { diam- } \\
\text { eter } \geq 300 \mu \mathrm{m}) \\
\text { PI }(\text { diame- } \\
\text { ter }<300 \mu \mathrm{m})\end{array}$ & $\mathrm{Pt} / \mathrm{PtIr} / \mathrm{SS}$ & $\begin{array}{l}\text { Proportional to substrate } \\
\text { material and nerve } \\
\text { size }\end{array}$ & $1-24$ & Not FDA approved \\
\hline Micro-leads & www.micro-leads.com & PI/Silicone & PtIr & $\begin{array}{l}250 \mu \mathrm{m} \\
\quad(\text { o.d. }=1 / 1.4 \mathrm{~mm})\end{array}$ & $\mathrm{B} / \mathrm{T}$ & Not FDA approved \\
\hline CorTec & cortec-neuro.com & Silicone & $\mathrm{Pt} / \mathrm{PtIr}^{\mathrm{a}}$ & $0.2-0.8 \mathrm{~mm}$-spiralcuff & $2-8$ & - \\
\hline Ardiem Medical, Inc. & $\begin{array}{l}\text { www.ardiemmedical. } \\
\text { com }\end{array}$ & Silicone & $\mathrm{Pt}$ & - & $1-4$ & Not FDA approved \\
\hline
\end{tabular}

$S S$ stainless steel, $P I$ polyimide, $B$ bipolar, $T$ tripolar

${ }^{\#}$ Optional coatings of sputtered IrOx, Pt black 
of the appropriate size and statistical power" (Fins et al. 2011); this is reserved for patients who suffer from rare or unusual conditions where there is an insufficient number of patients with the condition to warrant a commercial interest in the treatment.

\section{Discussion}

Whilst there appears to be significant strides in developing implantable technology, there has yet to be a single method which can be used confidently for interfacing with the PNS. For example, Hoffmann et al. state that there has yet be a standardised method for quality electrical encapsulation, though composite bilayers are offering a potential solution (Bettinger 2018; Hoffmann et al. 2006). Despite this, PNS implants have been used for clinical applications, particularly for direct stimulation of pain sites of those suffering chronic pain; consequently, there have been several reviews highlighting the failures of these implantable technologies (Eldabe et al. 2015). As such, we cannot yet guarantee the lifetime or stability of a neural implant and therefore remain open to external interfacing with the PNS via cutaneous electrodes. This allows easy replacement of faulty components as well as recalibration of the interface to ensure optimum functionality of technology. Furthermore, this drops the FDA criteria of the interface devices from a Class III to Class I/II, making the technologies not only easier to market, but also to clinically test and develop prosthetic devices. This has fostered a faster development of myoelectric prosthetic control using these bio-interfaces resulting in strides in this area of research as engineers can develop the technologies without clinical support. Despite this, there remains a commercial and clinical lag behind academic research impact highlighted by Jiang et al. as being owed to a loss of development in system resilience to "changes in EMG signal characteristics" as well as failing to "integrate other sensor modalities to allow complex actions" of prosthetic control (Jiang et al. 2012).

Similarly, there is a technological lag between academic and commercially developed PNS electrodes, with the latter seeing slow incremental changes adopted from academic studies. Further, the lack of FDA approval for these technologies will hinder the adoption of these interface technologies into novel applications benefitting patients. This has driven significant strides in applying prosthetic technologies utilising indirect sensory feedback methods which are currently lagging PNS neural interface technology in their specificity to record and stimulate individual neurons.

Myoelectric control will remain the foremost method for bio-interfacing the body with a smart prosthetic but will eventually lag implantable technologies as the research community continues to rapidly address the many complexities of long-term chronic implants for stable bi-directional electrical interfaces with the nerves.

OpenAccess This article is distributed under the terms of the Creative Commons Attribution 4.0 International License (http://creativeco mmons.org/licenses/by/4.0/), which permits unrestricted use, distribution, and reproduction in any medium, provided you give appropriate credit to the original author(s) and the source, provide a link to the Creative Commons license, and indicate if changes were made.

\section{References}

Adewole, D.O., et al.: The evolution of neuroprosthetic interfaces. Crit. Rev. Biomed. Eng. 44(1-02), 123-152 (2016)

Agarwal, K., Jegadeesan, R., Guo, Y.-X., Thakor, N.V.: Wireless power transfer strategies for implantable bioelectronics. IEEE Rev. Biomed. Eng. 10, 136-161 (2017)

Amsuess, S., et al.: Context-Dependent Upper Limb Prosthesis Control for Natural and Robust Use. IEEE Trans. Neural Syst. Rehabil. Eng. 24(7), 744-753 (2016)

Angeli, C.A., et al.: Recovery of over-ground walking after chronic motor complete spinal cord injury. N. Engl. J. Med. 379(13), 1244-1250 (2018)

Barrese, J.C., et al.: Failure mode analysis of silicon-based intracortical microelectrode arrays in non-human primates. J. Neural Eng. 10(6), 066014 (2013)

Ben-Menachem, E.: Vagus-nerve stimulation for the treatment of epilepsy. Lancet Neurol. 1(8), 477-482 (2002)

Bettinger, C.J.: Recent advances in materials and flexible electronics for peripheral nerve interfaces. Bioelectron. Med. 4(1), 6 (2018)

Boretius, T., et al.: A transverse intrafascicular multichannel electrode (TIME) to interface with the peripheral nerve. Biosens. Bioelectron. 26(1), 62-69 (2010)

Brindley, G.S.: The first 500 patients with sacral anterior root stimulator implants: general description. Spinal Cord 32(12), 795-805 (1994)

Brindley, G.S., Polkey, C.E., Rushton, D.N.: Sacral anterior root stimulators for bladder control in paraplegia. Spinal Cord 20(6), 365-381 (1982)

Charkhkar, H., Shell, C.E., Marasco, P.D., Pinault, G.J., Tyler, D.J., Triolo, R.J.: High-density peripheral nerve cuffs restore natural sensation to individuals with lower-limb amputations. J. Neural Eng. 15(5), 056002 (2018)

Cheung, K.C.: Implantable microscale neural interfaces. Biomed. Microdevices 9(6), 923-938 (2007)

Cobo, A.M., Boyajian, B., Larson, C., Schotten, K., Pikov, V., Meng, E.: A parylene cuff electrode for peripheral nerve recording and drug delivery. In: 2017 IEEE 30th International Conference on Micro Electro Mechanical Systems (MEMS), pp. 506-509 (2017)

Cogan, S.F.: Neural Stimulation and Recording Electrodes. Annu. Rev. Biomed. Eng. 10(1), 275-309 (2008)

Cui, X., Martin, D.C.: Fuzzy gold electrodes for lowering impedance and improving adhesion with electrodeposited conducting polymer films. Sens. Actuators A Phys. 103(3), 384-394 (2003)

Dhillon, G.S., Lawrence, S.M., Hutchinson, D.T., Horch, K.W.: Residual function in peripheral nerve stumps of amputees: implications for neural control of artificial limbs. J. Hand Surg. Am. 29(4), 605-615 (2004)

Eldabe, S., Buchser, E., Duarte, R.V.: Complications of spinal cord stimulation and peripheral nerve stimulation techniques: a review of the literature. Pain Med. 17(2), pnv025 (2015) 
Ereifej, E.S., et al.: Nanopatterning effects on astrocyte reactivity. J. Biomed. Mater. Res. Part A 101A(6), 1743-1757 (2013a)

Ereifej, E.S., Cheng, M.M.-C., Mao, G., VandeVord, P.J.: Examining the inflammatory response to nanopatterned polydimethylsiloxane using organotypic brain slice methods. J. Neurosci. Methods 217(1-2), 17-25 (2013b)

Fins, J.J., et al.: Misuse of the FDA's humanitarian device exemption in deep brain stimulation for obsessive-compulsive disorder. Health Aff. 30(2), 302-311 (2011)

Freeberg, M.J., Stone, M.A., Triolo, R.J., Tyler, D.J.: The design of and chronic tissue response to a composite nerve electrode with patterned stiffness. J. Neural Eng. 14(3), 036022 (2017)

Geddes, L.A., Roeder, R.: Criteria for the Selection of Materials for Implanted Electrodes. Ann. Biomed. Eng. 31(7), 879-890 (2003)

Gesslbauer, B., Hruby, L.A., Roche, A.D., Farina, D., Blumer, R., Aszmann, O.C.: Axonal components of nerves innervating the human arm. Ann. Neurol. 82(3), 396-408 (2017)

Ghafoor, U., Kim, S., Hong, K.-S.: Selectivity and longevity of peripheral-nerve and machine interfaces: a Review. Front. Neurorobot. 11, 59 (2017)

Gill, M.L., et al.: Neuromodulation of lumbosacral spinal networks enables independent stepping after complete paraplegia. Nat. Med. 24, 1677 (2018)

Graczyk, E.L., Resnik, L., Schiefer, M.A., Schmitt, M.S., Tyler, D.J.: Home use of a neural-connected sensory prosthesis provides the functional and psychosocial experience of having a hand again. Sci. Rep. 8(1), 9866 (2018)

Guo, L.: The pursuit of chronically reliable neural interfaces: a materials perspective. Front. Neurosci. 10, 599 (2016)

Haugland, M.: A flexible method for fabrication of nerve cuff electrodes. In: Proceedings of 18th Annual International Conference of the IEEE Engineering in Medicine and Biology Society, Amsterdam, Netherlands, 31 October-3 November 1996, vol. 1, pp. 359-360 (1996). https://doi.org/10.1109/IEMBS.1996.656992

Hoffmann, K.P., Koch, K.P., Doerge, T., Micera, S.: New technologies in manufacturing of different implantable microelectrodes as an interface to the peripheral nervous system. In: The First IEEE/ RAS-EMBS International Conference on Biomedical Robotics and Biomechatronics. BioRob 2006., pp. 414-419 (2006)

Jiang, N., Dosen, S., Muller, K.-R., Farina, D.: Myoelectric control of artificial limbs-is there a need to change focus? [In the Spotlight]. IEEE Signal Process. Mag. 29(5), 150-152 (2012)

Joung, Y.-H.: Development of implantable medical devices: from an engineering perspective. Int. Neurourol. J. 17(3), 98-106 (2013)

Koo, J., et al.: Wireless bioresorbable electronic system enables sustained nonpharmacological neuroregenerative therapy. Nat. Med. 24, 1 (2018)

Lago, N., Ceballos, D., Rodŕguez, F.J., Stieglitz, T., Navarro, X.: Long term assessment of axonal regeneration through polyimide regenerative electrodes to interface the peripheral nerve. Biomaterials 26(14), 2021-2031 (2005)

Lee, S., et al.: Toward bioelectronic medicine-neuromodulation of small peripheral nerves using flexible neural clip. Adv. Sci. 4(11), 1700149 (2017a)

Lee, S., et al.: Selective stimulation and neural recording on peripheral nerves using flexible split ring electrodes. Sens Actuators B Chem. 242, 1165-1170 (2017b)

Lissandrello, C.A., et al.: A micro-scale printable nanoclip for electrical stimulation and recording in small nerves. J. Neural Eng. 14(3), 036006 (2017)

Lyons, G.M., Sinkjaer, T., Burridge, J.H., Wilcox, D.J.: A review of portable FES-based neural orthoses for the correction of drop foot. IEEE Trans. Neural Syst. Rehabilit. Eng. 10(4), 260-279 (2002)

McDonnall, D., Clark, G.A., Normann, R.A.: Interleaved, multisite electrical stimulation of cat sciatic nerve produces fatigue-resistant, ripple-free motor responses. IEEE Trans. Neural Syst. Rehabil. Eng. 12(2), 208-215 (2004)

Mishra, S.: Electroceuticals in medicine-the brave new future. Indian Heart J. 69(5), 685-686 (2017)

Mitcheson, P.D.: Energy harvesting for human wearable and implantable bio-sensors. In 2010 Annual International Conference of the IEEE Engineering in Medicine and Biology, pp. 3432-3436 (2010)

Naples, G.G., Mortimer, J.T., Scheiner, A., Sweeney, J.D.: A spiral nerve cuff electrode for peripheral nerve stimulation. IEEE Trans. Biomed. Eng. 35(11), 905-916 (1988)

Navarro, X., Krueger, T.B., Lago, N., Micera, S., Stieglitz, T., Dario, P.: A critical review of interfaces with the peripheral nervous system for the control of neuroprostheses and hybrid bionic systems. J. Peripher. Nerv. Syst. 10(3), 229-258 (2005)

Ordonez, J.S. et al.: Cuff electrodes for very small diameter nervesprototyping and first recordings in vivo. In: 2014 36th Annual International Conference of the IEEE Engineering in Medicine and Biology Society, EMBC 2014, pp. 6846-6849 (2014)

Pannek, J., Grigoleit, U., Hinkel, A.: Bacterial contamination of test stimulation leads during percutaneous nerve stimulation. Urology 65(6), 1096-1098 (2005)

Raspopovic, S., et al.: Restoring natural sensory feedback in realtime bidirectional hand prostheses. Sci. Transl. Med. 6(222), 222ra19 (2014)

Roche, A.D., Rehbaum, H., Farina, D., Aszmann, O.C.: Prosthetic myoelectric control strategies: a clinical perspective. Curr. Surg. Rep. 2(3), 44 (2014)

Rossini, P.M., et al.: Double nerve intraneural interface implant on a human amputee for robotic hand control. Clin. Neurophysiol. 121(5), 777-783 (2010)

Russell, C., Kissane, R.W.P., Steenson, D.P., Chakrabarty, S.: A novel simplistic fabrication technique for cranial epidural electrodes for chronic recording and stimulation in rats. J. Neurosci. Methods 311, 239-242 (2019). https://doi.org/10.1016/j.jneum eth.2018.10.036

Rutten, W.L.C.: Selective electrical interfaces with the nervous system. Annu. Rev. Biomed. Eng. 4(1), 407-452 (2002)

Schiefer, M., Tan, D., Sidek, S.M., Tyler, D.J.: Sensory feedback by peripheral nerve stimulation improves task performance in individuals with upper limb loss using a myoelectric prosthesis. J. Neural Eng. 13(1), 016001 (2016)

Schlie-Wolter, S., Deiwick, A., Fadeeva, E., Paasche, G., Lenarz, T., Chichkov, B.N.: Topography and Coating of Platinum Improve the Electrochemical Properties and Neuronal Guidance. ACS Appl. Mater. Interfaces. 5(3), 1070-1077 (2013)

Schultz, A.E., Kuiken, T.A.: Neural interfaces for control of upper limb prostheses: the state of the art and future possibilities. PM\&R 3(1), 55-67 (2011)

Schwartz, A.B., Cui, X.T., Weber, D.J., Moran, D.W.: Brain-Controlled Interfaces: movement Restoration with Neural Prosthetics. Neuron 52(1), 205-220 (2006)

Seddon, H.J.: A classification of nerve injuries. Br. Med. J. 2(4260), 237-239 (1942)

Stein, R.B., Charles, D., Davis, L., Jhamandas, J., Mannard, A., Nichols, T.R.: Principles underlying new methods for chronic neural recording. Can. J. Neurol. Sci. 2(3), 235-244 (1975)

Stieglitz, T., Beutel, H., Schuettler, M., Meyer, J.-U.: Micromachined, polyimide-based devices for flexible neural interfaces. Biomed. Microdevices 2(4), 283-294 (2000)

Tan, D.W., Schiefer, M.A., Keith, M.W., Anderson, J.R., Tyler, J., Tyler, D.J.: A neural interface provides long-term stable natural touch perception. Sci. Transl. Med. 6(257), 257ra138 (2014)

Teshome, A.K., Kibret, B., Lai, D.: A review of implant communication technology in WBAN : progresses and challenges. 
IEEE Rev. Biomed. Eng. (2018). https://doi.org/10.1109/ RBME.2018.2848228

Tipnis, N.P., Burgess, D.J.: Sterilization of implantable polymerbased medical devices: a review. Int. J. Pharm. 544(2), 455-460 (2018)

Tyler, D.J., Durand, D.M.: A slowly penetrating interfascicular nerve electrode for selective activation of peripheral nerves. IEEE Trans. Rehabilit. Eng. 5(1), 51-61 (1997)

Tyler, D.J., Durand, D.M.: Functionally selective peripheral nerve stimulation with a flat interface nerve electrode. IEEE Trans. Neural Syst. Rehabilit. Eng. 10(4), 294-303 (2002)

Wagner, F.B., et al.: Targeted neurotechnology restores walking in humans with spinal cord injury. Nature 563(7729), 65-71 (2018)

Wilson, B.S., Finley, C.C., Lawson, D.T., Wolford, R.D., Eddington, D.K., Rabinowitz, W.M.: Better speech recognition with cochlear implants. Nature 352(6332), 236-238 (1991)

Xiang, Z., et al.: Progress of flexible electronics in neural interfacinga self-adaptive non-invasive neural ribbon electrode for small nerves recording. Adv. Mater. 28(22), 4472-4479 (2016)

Yoshida, K., Pellinen, D., Pivin, D., Rousche, P., Kipke, D.: Development of the thin-film longitudinal intra-fascicular electrode. In: Center for Sensory-Motor Interaction (SMI), Department of Health Science and Technology, Aalborg University, pp. 279-281 (2000)

Yu, H., Xiong, W., Zhang, H., Wang, W., Li, Z.: A parylene self-locking cuff electrode for peripheral nerve stimulation and recording. J. Microelectromechanical Syst. 23(5), 1025-1035 (2014)

Publisher's Note Springer Nature remains neutral with regard to jurisdictional claims in published maps and institutional affiliations.

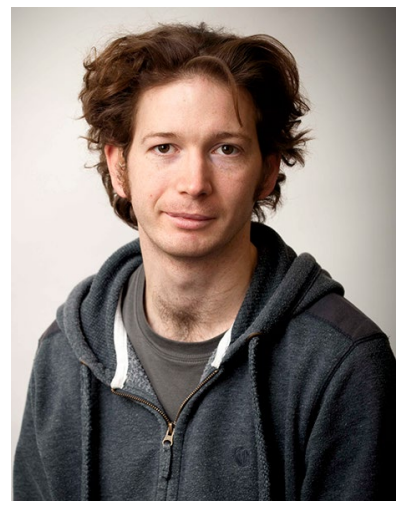

Christopher Russell received his BEng in Electronic and Electrical Engineering from the University of Leeds before completing a $\mathrm{PhD}$ in on-chip THz-time domain spectroscopy systems at the Pollard Institute within the same university. He has subsequently realigned his post-doctoral studies to neuroscience applications; designing and fabricating neural interface tools for exploratory sciences. He has an active interest in healthcare technologies exploring and engineering the bio-interaction between implantable and wearable technologies for ensuring longevity and specificity of treatments.

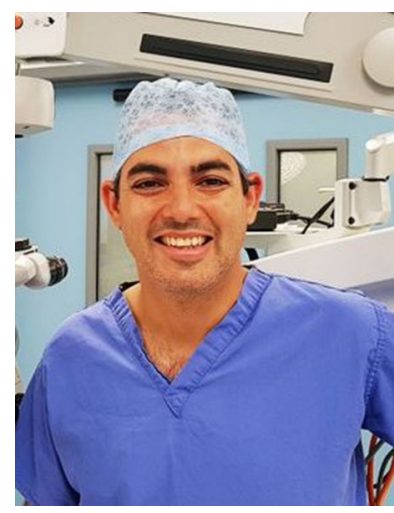

Aidan D. Roche has been developing his research interest into bionic applications to clinical medicine over the past 15 years. After receiving his BEng in Biomedical Engineering, he began his training in The Neural Engineering Centre for Artificial Limbs of the Rehabilitation Institute of Chicago, investigating intramuscular electrodes for prosthetic control. After this, he undertook his $\mathrm{PhD}$ within the Department of Bioengineering of Imperial College London, where he identified selective sensorimotor cortical networks responsible for fine human hand movements. Dr Roche then completed his medical training at Imperial, before undertaking clinical academic training within the UK's National Health Service, specialising in Plastic \& Reconstructive Surgery. His initial postdoctoral training was completed in the Bionic Reconstruction Lab of the Medical University of Vienna, where he contributed to the implementation of bionic systems in real world clinical applications. Dr Roche continues his interest in the field of Bionic Medicine as a Clinical Lecturer at The University of Edinburgh.

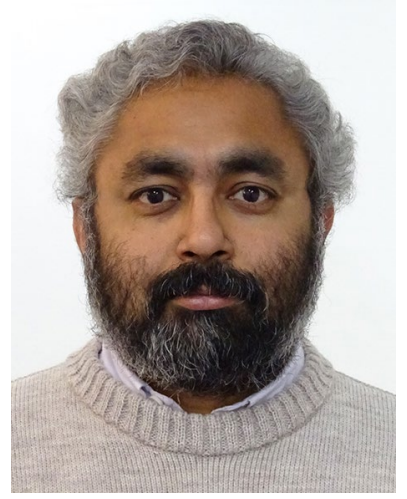

Samit Chakrabarty has been studying the role of spinal circuits in execution of motor tasks, their modulation by peripheral sensory and descending inputs from the brain, focussing on the plastic changes that the system undertakes during development or disease. After receiving his $\mathrm{BSc}$ in Zoology, Biochemistry from St Xavier's College, Mumbai he pursued a $\mathrm{PhD}$ in Neurophysiology of the mammalian spinal cord at the University of Cambridge, UK. This was then followed by postdoctoral training at Columbia University, NYC and University of Manitoba, Winnipeg. He has since moved to University of Leeds as an academic researcher and is active in the field of sensory and motor control, rehabilitation and use of technology to both study and better the lives of those with maladies affecting sensory and motor function. 